\title{
Mucormicosis en Pediatría
}

W M PÍA SPALLONI M., ANA CHÁVEZ P., CARMEN L AVILÉS L. y JOSÉ COFRÉ G.

\section{Mucormycosis in Pediatrics}

Mucormycosis is an infrequent infection caused by opportunistic fungi belonging to the Mucorales order; Mucoraceae family, whose characteristics are vascular invasion by hyphae, which determine thrombosis and tissue infarction. In generally affects patients with underlying diseases and produces serious invasive and often fulminant infections. Some of the risk factors leading to mucormycosis are diabetic ketoacidosis, immunosuppressive therapy, leukemia and lymphomas with prolonged neutropenia. Various clinical presentations are described, relating to anatomical site involved, being the rhinocerebral site, the most frequent one, specially in diabetic patients. Although this infection has a high morbidity and mortality, its prognosis has improved the last years, relating to therapeutic measures like correction of predisposing conditions, use of amphotericin B and early aggressive surgery. It is very important to suspect this infection in patients with predisposing conditions so an early diagnosis can be made.

Key words: Mucormycosis; Zygomycosis; Pediatrics; Diagnosis; Treatment

\section{Introducción}

También llamada phycomicosis o zigomicosis, (aunque este segundo término es más amplio y engloba además a infecciones causadas por Entomophtorales), es una afección causada por hongos saprófítos aeróbicos, generalmente no patógenos para el hospedero inmunocompetente ${ }^{1,2}$. El desarrollo de enfermedad depende del estado inmune del huésped; los macrófagos y neutrófilos parecen ser el componente primario de la respuesta inmune contra estos microorganismos, previniendo la germinación de las esporas inhaladas ${ }^{3}$.

El sello de la mucormicosis es la invasión vascular por hifas que conduce a la trombosis arterial, infarto secundario, trombosis venosa y hemorragia consecuente. Las razones de la afinidad del hongo por el tejido vascular son desconocidas y materia de especulación ${ }^{2,5}$.

\section{Etiología}

Todos los agentes de mucormicosis pertenecen al orden Mucorales. Los agentes etiológicos descritos en el hombre son Rhizopus oryzae, Rhizopus microsporus, Cunninghamella bertholletiae, Saksenaea vasiformis, Mucor circinelloides, Mucor ramosissimus, Absidia corymbifera, Rhizomucor pusillus, Apophysomyces elegans, Cokeromyces recurvatus y Syncephalastrum racemosum. ${ }^{1-4}$.

Los hongos del Orden Mucorales son microorganismos no fastidiosos que crecen en un rango de temperatura de 25 a $55^{\circ} \mathrm{C}$, aeróbicos y demoran 2 a 5 días en formar colonias visibles a ojo desnudo. Las colonias tienen aspecto lanudo, a menudo parecen tenues puntos negros. Las especies de estos géneros desarrollan estolones, rizoides (en Rhizopus, Absidia y Rhizomucor) y el esporangio contiene en su extremo miriadas de esporangiosporas de 3-6 $\mathrm{mm}$ de diámetro cada una, que sirven como la forma de diseminación al medioambiente. La observación directa del tejido extraído, tratado con $\mathrm{KOH}$ al $10 \%$, permite apreciar formas irregulares, hifas características largas y con ancho de 10 a $30 \mathrm{~m} \mu$, no septadas, que adoptan a menudo formas curvas o de cintas ramificadas en ángulo recto (Figura 1), disposición que permite el libre flujo de núcleos y

Hospital Militar del General Luis Felipe Brieba Arán (WMPSM)

Hospital Dr. Exequiel González Cortés (ACP)

Hospital San Borja Arriarán (CLAL)

Hospital Luis Calvo Mackenna (JCG)

Recibido: $1^{\circ}$ septiembre 2003

Aceptado: 14 octubre 2003 


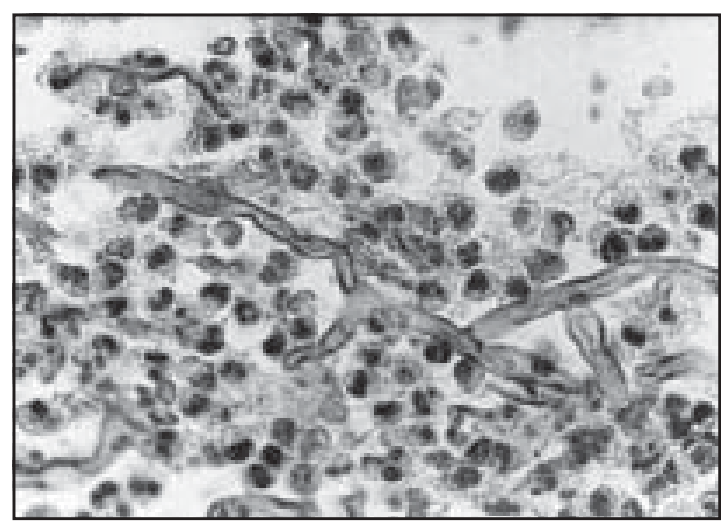

Figura 1.

organelos citoplasmáticos; por esta razón se desarrollan rápidamente en los medios de cultivo habituales ${ }^{3,4}$. Las muestras clínicas deben ser sembradas en agar Sabouraud con antibacterianos para inhibir la flora bacteriana acompañante, sin ciclohexamida, e incubadas a dos temperaturas, ambiental y a $37^{\circ} \mathrm{C}^{4}$. El fierro actúa como un factor de multiplicación in vitro, tal como sucede con muchos microorganismos.

En mucormicosis los agentes más frecuentemente aislados son miembros de la familia Mucoraceae (Tabla 1), específicamente Rhizopus, Rhizomucor y Absidia y dentro del género Rhizopus, $R$. oryzae, seguido de $R$. microsporus var. rhizopodiformis; en pacientes pediátricos los agentes etiológicos identificados han sido fundamentalmente Rhizopus sp y esporádicamente Absidia $\mathrm{sp}^{2,5,6}$.

Tabla 1. Clasificación de los Zygomycetes

Zygomycetes (clase)

$\Downarrow$

Mucorales (orden)

$\Downarrow$

Mucoraceae (familia)

$\Downarrow$

Rhizopus

Absidia

Rhizomucor

Mucor

Apophysomyces

\section{Epidemiología}

Son microorganismos ubicuos y la exposición a las esporas de estos hongos es inevitable; la baja frecuencia de infecciones observadas en la práctica clínica revela su baja virulencia ${ }^{7}$. Las Mucoraceae no forman parte de la flora microbiana residente del hombre, ocasionalmente se cultivan desde la piel o mucosas de pacientes hospitalizados ${ }^{8}$.

La mucormicosis, tiene una amplia distribución geográfica ya que los agentes causales son ubicuos encontrándose en vegetales en descomposición, semillas y frutas, pan añejo, suelo, excretas animales, o en cualquier sustrato que contenga carbohidratos, donde esporula abundantemente siendo transportada a distancia en el aire $^{3,4}$; han sido identificados incluso en el medio ambiente hospitalario lo que representa, al igual que Aspergillus sp, una amenaza para la población de pacientes inmunocomprometidos ${ }^{8,9}$. Las esporangiosporas pueden ser vehiculizadas en el medio hopitalario por corrientes de aire o por objetos en contacto directo con los pacientes, hay varios reportes de colonización de piel y mucosas y de enfermedad por el uso de bajalenguas de madera contaminados ${ }^{10}$.

La mucormicosis debe entenderse como una enfermedad de muy baja frecuencia. En adultos ésta ha sido estimada 10 veces menor que la aspergilosis, aunque en la medida que aumentan los hospederos propicios para su proliferación, ya se observa una mayor incidencia ${ }^{10}$. La literatura médica nacional es escasísima acerca de esta patología; en los últimos 20 años encontramos: en 1985 dos casos de mucormicosis orbitaria y cerebral en adultos comunicados por J. Cox et $\mathrm{al}^{11}$ y un caso de mucormicosis orbitaria en un adulto diabético, por F. Villarroel ${ }^{12}$; en 1987 un reporte anátomo-clínico de 17 casos en adultos por D. Oddó y cols y que abarca 10 años de pesquisa de la enfermedad en servicios de Anatomía Patológica en hospitales de la ciudad de Santiago causando el deceso en 15 de ellos ${ }^{13}$; en 1991 un caso de mucormicosis pulmonar en paciente adulto inmunocomprometido, publicado por R. Gacitúa et $\mathrm{al}^{14}$; en 1999 M. Bravo et al comunicaron 4 casos de mucormicosis rinocerebral en adultos ${ }^{15}$; en 2001 P. García y cols comunicaron su experiencia en dos pacientes adultos ensayando una técnica diagnóstica -blanco de calcoflúor- en esta afección ${ }^{16}$; en 2003 un caso de mucormicosis rinosinusal en un adulto diabético reportado por H. Bertin. ${ }^{17}$

No hay menciones previas en la literatura médica chilena de casos pediátricos. En este núme- 
ro de la revista los autores de esta revisión presentamos por separado cuatro casos pediátricos ocurridos en los últimos 6 años ${ }^{18-21}$.

\section{Patogenia}

La infección se adquiere por inhalación de esporas, las que colonizan los senos paranasales y el nasofarinx; ocasionalmente se puede adquirir por ingestión inadvertida o inoculación cutánea de esporas. No se ha documentado transmisión persona a persona ${ }^{3}$. Estos hongos muestran alta afinidad por las zonas aireadas del cuerpo, por su metabolismo aeróbico; ello explicaría, en parte, su predilección por el tracto respiratorio y su afinidad por las arterias.

Entre los factores que aparecen constantemente citados como favorecedores de la enfermedad están: la acidosis metabólica, la cetoacidosis diabética y el uso de desferoxamina; condiciones todas en que se produce un incremento del ión $\mathrm{Fe}^{+++}$libre en el plasma que, como fuera señalado, actúa como factor estimulante del crecimiento y multiplicación de los hongos de esta familia. En la acidosis metabólica y diabetes descompensada, la disminución del $\mathrm{pH}$ permite la liberación del $\mathrm{Fe}^{+++}$acoplado a la transferrina plasmática. ${ }^{22 .}$ También se ha invocado para explicar la mayor incidencia de esta enfermedad durante episodios de cetoacidosis diabética, la presencia de una enzima ketoreductasa en el hongo, enzima que permitiría la utilización de los cuerpos cetónicos circulantes durante la descompensación diabética. ${ }^{22}$ A su vez, la desferoxamina, utilizada como agente quelante del $\mathrm{Fe}^{+++}$en sujetos que tienen depósitos tisulares patológicos del metal (por ejemplo pacientes con hemocromatosis, politransfundidos o hemodializados), desprende y captura este $\mathrm{Fe}^{+++}$para eliminarlo por vía fecal y urinaria, lo que expone al paciente colonizado a desarrollar enfermedad. Además el hongo es capaz de utilizar la desferoxamina como un sideróforo en su propio beneficio incrementando su viabilidad $^{23,24}$. No se han descrito toxinas propias para este grupo de hongos.

La capacidad fagocítica de neutrófilos y macrófagos es un factor defensivo primordial contra la enfermedad; así, en personas con neutropenia prolongada se facilita la invasión de mucosas y tejidos. Los macrófagos alveolares inhiben también la esporulación habiéndose comprobado que en los pacientes con cetoacidosis diabética este mecanismo defensivo está inhibido ${ }^{25}$. Por otra parte, en sujetos con deficiencias del sistema inmune celular (por ejemplo linfomas), existe mayor susceptibilidad que en la población general para contraer mucormicosis; no se conoce bien cómo la inmunidad celular protege contra la invasión por estos hongos.

La mucormicosis ocasiona inflamación aguda o crónica de los tejidos. Las hifas invaden los tejidos y especialmente las paredes de los vasos sanguíneos y su lumen "en busca de oxígeno", con propensión a producir trombosis y necrosis; además, del foco primario se pueden desprender émbolos sépticos hacia cualquier parte del organismo $^{1,2}$. En la forma rinocerebral la enfermedad puede progresar hacia el cerebro a lo largo de las raíces nerviosas, o por extensión directa, a través del fondo de la órbita. El traumatismo de piel y mucosas es un factor casi constante en los reportes de infecciones cutáneas, de la vía respiratoria alta e intestinal.

Otras enfermedades y/o condiciones que se han asociado con mucormicosis son leucemia en fase de neutropenia prolongada, linfoma y síndrome mielodisplásico, tratamiento inmunosupresor, terapia corticoesteroidal, trasplante de órganos sólidos y de precursores hematopoyéticos, pacientes quemados (en los que mucor generalmente compromete la piel), prematurez e infección por $\mathrm{VIH}^{1,2,7,26,27}$.

\section{Manifestaciones clínicas}

Se describen seis formas clínicas de mucormicosis: cutánea, gastrointestinal, pulmonar, rinocerebral, diseminada y una forma miscelánea, que engloba localizaciones como endocarditis, miocarditis, meningitis, absceso cerebral, endoftalmitis, cistitis, peritonitis, osteomielitis, artritis, miositis y pielonefritis ${ }^{1-3}$.

En niños se han descrito principalmente las formas cutánea e intestinal en neonatos y las formas pulmonar y rinocerebral en pacientes diabéticos e inmunocomprometidos ${ }^{2,26,27}$.

\section{Mucormicosis cutánea primaria}

Bajo el concepto de primario se excluyen las infecciones metastásicas de piel.

Se han descrito dos formas de presentación: superficial y gangrenosa o subcutánea.

Mucormicosis superficial ${ }^{26}$. Se presenta en pacientes inmunocompetentes, en quienes un traumatismo facilita la penetración del hongo a través de la piel, donde se replica en forma lenta causando un área de necrosis relativamente circunscrita; se inicia como vesículas o pústulas que pronto se transforman en úlceras o escaras. Se ha descrito en neonatos con venopunciones, catéteres 
umbilicales intravasculares, en asociación con uso de monitores cutáneos, en pacientes quemados, heridas operatorias e intubación endotraqueal prolongada. En estos casos la humedad local y el calor ambiental propio del medio hospitalario favorecen la proliferación del hongo.

Mucormicosis gangrenosa o subcutánea. Se inicia en forma semejante a la anterior pero la ulceración y la extensión regional son rápidas comprometiendo músculos, tendones y hueso; pueden diseminarse por vía sanguínea teniendo un curso fulminante. Se ha descrito preferentemente en pacientes inmunocomprometidos y diabéticos ${ }^{1,27,28}$.

\section{Mucormicosis gastrointestinal}

Se presenta en pacientes desnutridos, aclorhídricos o inmunocomprometidos. Puede manifestarse como un cuadro de abdomen agudo de inicio insidioso y en el examen físico o por imagenología percibirse una masa abdominal; se ha descrito invasión de otras vísceras y perforación intestinal con peritonitis secundaria. La mucormicosis gastrointestinal debería plantearse en neonatos con enterocolitis necrosante que no mejora con las medidas médicas y quirúrgicas habituales ${ }^{27}$.

\section{Mucormicosis pulmonar}

Es más frecuente en pacientes con las siguientes condiciones clínicas: inmunocomprometidos, neoplasias hematológicas o trasplantes de precursores hematopoyéticos y neutropenia severa, corticoterapia prolongada ${ }^{7,29}$. Menos frecuentemente se ha reportado en pacientes diabéticos, sometidos a trasplante renal, infectados por VIH,

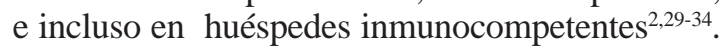
La zigomicosis pulmonar puede ocurrir también como parte de una infección diseminada y raras veces después de una enfermedad rinocerebral ${ }^{1,34}$. Se produce por inhalación de esporas del medio ambiente, el hongo suele colonizar inicialmente las cavidades perinasales en forma silenciosa y luego descender hacia el tracto respiratorio inferior. Allí daña las paredes bronquiales, el tejido peribronquial y provoca trombosis e infarto pulmonar, determinando extensa necrosis e invasión del tejido vascular, infartos con diseminación al resto de los pulmones y tejidos extra pulmonares; puede invadir por vecindad al pericardio, miocardio, vena cava superior y diafragma, lo que explica la hemoptisis masiva, manifestación característica de esta enfermedad ${ }^{14,35,36}$.

Las manifestaciones clínicas son inespecíficas, fiebre moderada, tos productiva, hemoptisis, disnea y dolor torácico.
Existe una amplia gama de presentaciones morfológicas: nódulo solitario, consolidación lobar, segmentaria, cavitaria o bronconeumonía, absceso pulmonar, mediastinitis fibrosante, obstrucción de vena cava superior y linfadenopatía cervical $^{37-39}$. La radiografía de tórax muestra infiltrados pulmonares o masas nodulares, que se localizan de preferencia en los lóbulos superiores $^{39}$. Puede también mostrar consolidación y lesiones cavitarias. La presencia del signo de "aire en creciente" (aire entre una lesión parenquimatosa radiodensa y tejido pulmonar normal) parece asociarse significativamente a un riesgo mayor de hemoptisis masiva. Las efusiones pleurales son infrecuentes, $(6-8 \%)^{39}$.

La TAC de tórax revela el "signo del halo" (baja atenuación alrededor del infiltrado) y reforzamiento en anillo después de la administración de medio de contraste y también el signo de "aire en creciente", que es altamente sugerente de patógeno fúngico oportunista ${ }^{40}$.

En pacientes neutropénicos la zigomicosis pulmonar es similar a la aspergilosis. Son frecuentes las infecciones mixtas, en 32 a $39 \%$ de los pacientes con mucormicosis pulmonar se ha descrito concomitancia con infecciones bacterianas o virales, cifra que puede elevarse hasta $83 \%$ en pacientes oncológicos.

El diagnóstico de zigomicosis pulmonar se basa frecuentemente en la morfología de los elementos micóticos debido al bajo rendimiento de los cultivos, siendo la muestra obtenida por fibrobroncoscopia con lavado broncoalveolar la más utilizada $^{41}$. El examen histopatológico de biopsias o piezas quirúrgicas confirma el diagnóstico en la mayoría de los casos y permite diferenciar zigomicosis de otras infecciones fúngicas ${ }^{1,2,7,39}$.

La letalidad en series que incluyen niños es alrededor de $50 \%$, teniendo mejor pronóstico los pacientes tratados en forma combinada médicoquirúrgica ${ }^{29,31,34}$.

\section{Mucormicosis rinocerebral}

En la población adulta es más frecuente en pacientes diabéticos mal controlados, con cetoacidosis $^{1,11,34}$. En niños la pesquisa de reportes en la literatura médica es escasísima y en su mayoría corresponden a pacientes con cáncer.

En esta localización se comprometen los vasos y nervios orbitarios y, eventualmente, los senos cavernosos. La infección se extiende a lo largo de las estructuras vasculares y neuronales e infiltra las paredes de los vasos sanguíneos. La infección horada las paredes óseas de las cavidades paranasales $(\mathrm{CPN})$, extendiéndose hacia la órbita y el área retro orbitaria, pudiendo compro- 
meter el cerebro ${ }^{1,42}$. Es común la invasión de nervios, vasos sanguíneos, cartílago, hueso y meninges y la trombosis por invasión directa del hongo. En etapas avanzadas de la infección se puede producir trombosis del seno cavernoso, arteria carótida y vena yugular ${ }^{1,2,42}$.

El término rinocerebral, que implica compromiso de senos paranasales, se emplea cuando se detecta compromiso de este espacio, haya ocurrido o no la invasión del SNC, por el eminente riesgo de que así ocurra.

Los síntomas iniciales son inespecíficos lo que dificulta el diagnóstico precoz ${ }^{1}$. Las formas más frecuentes de presentación son: dolor de la cara (maxilar, periorbitario o retro orbitario), rinorrea purulenta, epistaxis e hipoestesia nasal; cefalea y letargia en casos avanzados. Son signos acompañantes la visión borrosa, diplopia, proptosis, amaurosis uni o bilateral, úlcera del paladar y convulsiones o alteración de conciencia ${ }^{1}$. Una vez instalado el cuadro, se describe una tríada clásica constituida por: ceguera, oftalmoplejía y celulitis periorbitaria ${ }^{1}$.

$\mathrm{Al}$ examen físico el paladar y los cornetes pueden tener un aspecto gris o eritematoso, compromiso que puede progresar hacia la formación de una masa necrótica o ulceración; pese a ello, la mucosa sangra muy escasamente por la trombosis vascular antes referida. El compromiso neurológico se expresa como parálisis de los nervios craneanos: II, III, IV y VII y compromiso de conciencia en grado variable, desde letargia a coma, causado por el edema cerebral y compromiso vascular ${ }^{1,2,42}$.

La radiografía de cavidades paranasales (CPN) revela velamiento de los senos, pudiendo evidenciarse un nivel hidroaéreo. La TAC confirma el compromiso sinusal y puede documentar la destrucción ósea e invasión de la órbita. Además puede revelar trombosis del seno cavernoso, cerebritis y edema cerebral. La RM muestra estas alteraciones en forma más precoz que la TAC teniendo mayor sensibilidad para definir el compromiso vascular. El LCR habitualmente es normal ${ }^{1}$.

Las complicaciones son: trombosis de vasos intracraneanos (seno cavernoso, arteria carótida interna, vena yugular); infarto y necrosis de tejido cerebral; secuelas estéticas post cirugía y neurológicas ${ }^{1,2,34,42}$.

\section{Diagnóstico diferencial en mucormicosis}

El diagnóstico diferencial de la mucormicosis cutánea superficial debe plantearse con lesiones necróticas bacterianas, principalmente infeccio- nes por Streptococcus pyogenes y Pseudomonas aeruginosa. Un elemento que aumenta la sospecha etiológica es su lenta progresión, en días, a diferencia de lo que ocurre en las lesiones de causa bacteriana que en pocas horas crecen y comprometen el estado general del paciente.

Mucormicosis rinocerebral es una entidad dramática y distintiva, que puede ser confundida con trombosis del seno cavernoso, celulitis orbitaria bacteriana, aspergilosis rinocerebral o pseudallescheriosis.

La mucormicosis pulmonar debe diferenciarse de neumonía por bacterias gram negativas, aspergilosis y pseudallescheriosis; para ello es necesario recurrir a métodos invasores de obtención de muestras: LBA o biopsia pulmonar .

La mucormicosis gástrica puede asemejarse a la candidiasis por su habilidad de crecer en la superficie de úlceras. Candida sp provoca esofagitis y no es frecuente que cause perforación de víscera ${ }^{2,34}$,

\section{Diagnóstico etiológico}

Los exámenes generales de laboratorio son inespecíficos y no permiten sospechar la etiología; contribuyen sí a definir y monitorizar los factores causales.

El diagnóstico de compromiso tisular se confirma al demostrar la presencia de hifas invadiendo tejidos en muestras obtenidas por biopsia quirúrgica $\mathrm{y}$, en caso de afectación de pulmones o hígado, mediante LBA y punción aspirativa respectivamente.

El examen microscópico de tejidos fijados puede efectuarse mediante tinciones de hematoxilinaeosina, PAS, o metenamina plata (GomoriGrocott). Se puede implementar un diagnóstico rápido con proyección clínica (diagnóstico intraoperatorio) mediante la tinción de blancocalcoflúor combinada con fluorescencia ${ }^{16}$. El diagnóstico diferencial morfo e histopatológico con otras especies de hongos filamentosos se detalla en la Tabla 2. Estos criterios permiten identificar una infección por hongos de la orden Mucoraceae, siendo importante su diferenciación con Aspergillus sp, de mayor prevalencia y con Fusarium $\mathrm{sp}$, hongo emergente en nuestro medio.

El cultivo desde el sitio de la lesión es útil, pero más lento que el informe histopatológico. El cultivo de senos paranasales puede ser positivo, sin embargo no confirma el diagnóstico, porque puede reflejar colonización. En la forma diseminada los hemocultivos y urocultivos son habitualmente negativos. 
Tabla 2. Morfología e histopatología de Zygomycetes y otros hongos filamentosos ${ }^{(1)}$

\begin{tabular}{lll}
\hline Morfología & Zygomycetes & Otros hongos filamentosos* \\
\hline Elemento fúngico & Hifas & Hifas \\
Características de las hifas & & \\
- Ancho & anchas $(15-20 \mu \mathrm{m})$ & estrechas $(4-5 \mu \mathrm{m})$ \\
- Septos & Aseptadas & Septos característicos \\
- Paredes & Irregulares & Paralelas \\
Ramificaciones & Generalmente perpendicular & Generalmente agudas \\
Blastoconidias & Ausente & Ausente \\
Esporulación & Generalmente ausente $* * *$ & Ausente $* *$ \\
Angioinvasión & Presente & Presente \\
\hline
\end{tabular}

* Incluye Aspergillus spp, Fusarium spp, Pseudallescheria boydii.

** Puede estar presente si espacio infectado comunica con aire.

*** Hay informes de formación de clamidoconidia en tejido en cuatro casos de zigomicosis.

(1): Adaptado de ref 28

Hacer el diagnóstico de especie es habitualmente difícil y hasta ahora no parecía tener mayor trascendencia clínica; sin embargo, existen diferencias significativas de susceptibilidad in vitro de los distintos géneros y especies de la familia Mucoraceae a los azoles, lo que podría traducirse dentro de los próximos años en importantes decisiones terapéuticas ${ }^{43}$.

Se ha intentado desarrollar técnicas de inmunodiagnóstico (inmunodifusión y EIA) que han resultado poco sensible, inespecíficas y falsamente positivas en pacientes con aspergilosis y candidiasis.

Se ha ensayado la detección del genoma de estos hongos mediante RPC; se espera que, a futuro, el mayor desarrollo de estas técnicas de biología molecular permitan un diagnóstico precoz impactando en un mejor pronóstico.

\section{Tratamiento}

Se basa en tres pilares fundamentales: tratamiento de la enfermedad de base, terapia antifúngica sistémica y cirugía ${ }^{1,2,34,44,45}$.

\section{Tratamiento de la enfermedad de base}

Deben modificarse las condiciones que favorecen esta infección, como la hiperglicemia y la cetoacidosis diabética, o suspender o disminuir al mínimo la terapia inmunosupresora. Se ha ensayado el uso de factores estimulantes de colonias, basado en que neutrófilos tratados con estos factores han mostrado in vitro mayor actividad contra Aspergillus, Candida y Mucor, por mejoría del estallido respiratorio ${ }^{46,47}$.

\section{Terapia antifúngica sistémica}

Anfotericina $B$ : con efecto fungistático para Mucor, es el único fármaco recomendado hoy en día. Su actividad in vitro sobre las distintas especies es uniforme, con $\mathrm{CIM}_{90}$ que oscilan entre 0,25 y $1 \mu \mathrm{g} / \mathrm{ml}$; al momento actual no hay fármacos que hayan superado su actividad in vitro ni que hayan sido tan ensayados en clínica como la anfotericina $\mathrm{B}^{43}$. Existe al menos un reporte anecdótico de resistencia in vitro de Rhizopus sp a anfotericina B relacionado con fracaso clínico, situación que parece ser, por ahora, excepcio$\mathrm{nal}^{48}$.

La respuesta terapéutica a anfotericina B deoxicolato está limitada por la deficiente capacidad inmune del hospedero, sus condiciones metabólicas locales de hipoxia y acidosis, y por la poca penetración del fármaco en un terreno con oclusión arterial y trombosis venosa. Sin embargo, la introducción de anfotericina $\mathrm{B}$ deoxicolato en el tratamiento de la mucormicosis mejoró sustancialmente el pronóstico de la enfermedad que, antes de ella y pese a la cirugía radical, era casi uniformemente fatal. Parfrey hizo notar la mejoría en la sobrevida de la enfermedad neurológica en adultos de 6 a $73 \%$, desde antes a después de la introducción del tratamiento con anfotericina $\mathrm{B}^{45}$.

Dosis de anfotericina B: en niños se cita reiteradamente una dosis total de $30 \mathrm{mg} / \mathrm{kg}$, que ha sido establecida en forma empírica dada la baja casuística de mucormicosis en esta edad, lo que ha impedido hacer estudios comparativos; se recomienda administrar entre 1,0 y $1,5 \mathrm{mg} / \mathrm{kg} /$ día. En adultos se ha relacionado un mejor pronóstico con una dosis total de 2 gramos de 
anfotericina B. El requisito para poner término a la terapia antifúngica es la resolución clínica/ imagenológica de las lesiones.

La adición a la anfotericina $\mathrm{B}$ de otros fármacos para obtener efecto sinérgico, es controvertido y no recomendado en la actualidad ${ }^{34}$.

La introducción al uso clínico de formas lipídicas y liposomales de anfotericina $\mathrm{B}$ ha permitido elevar sus dosis a 3,5 y hasta $10 \mathrm{mg} / \mathrm{kg} / \mathrm{día}$ aumentando su concentración tisular, alcanzando antes una mayor impregnación del organismo y mejorando la tolerancia del fármaco. La experiencia publicada con estas formas de anfotericina $\mathrm{B}$ es mayor en adultos y escasamente encontramos un caso de mucormicosis en pediatría tratado exitosamente con anfotericina B complejo lipídico (ABLC) ${ }^{49}$.

Azoles. En general, tienen menor actividad sobre mucor que la anfotericina B. En evaluaciones recientes QN Sun et al y E. Dannaoui et al, coinciden en que in vitro la mayor actividad la tienen posaconazol con $\mathrm{CIM}_{90}$ de $1,0 \mu \mathrm{g} / \mathrm{ml}$ (rango $0,06-8,0 \mu \mathrm{g} / \mathrm{ml}) \mathrm{y}$, en menor grado, itraconazol con $\mathrm{CIM}_{90}$ de $8 \mu \mathrm{g} / \mathrm{ml}$ (rango 0,03-32,0 $\mu \mathrm{g} / \mathrm{ml}$ ) mientras que voriconazol y fluconazol son definitivamente inactivos presentando $\mathrm{CIM}_{90}$ elevadí$\operatorname{simas}^{50,51}$.

De esta mediciones in vitro se desprende que Absidia sp tiene la mayor susceptibilidad a posaconazol e itraconazol y que Rhizomucor sp tiene la máxima refractariedad; de aquí surge el interés terapéutico en identificar el género y especie de Mucor en cada paciente ${ }^{51}$.

Hay contados reportes hasta ahora, en la literatura médica, del empleo clínico de posaconazol en mucormicosis lo que no permite recomendar aún su indicación asociado a anfotericina $\mathrm{B}$ y menos, en su reemplazo. Recientemente se describió un adulto diabético, sometido a trasplante cardiaco y renal simultáneos, que desarrolló una infección mediastínica por Rhizopus sp refractaria a anfotericina $B$ en el que se ensayó posaconazol como monoterapia, logrando su curación ${ }^{52,53}$.

Equinocandinas: anidulafungin, caspofungin, y micafungin, constituyen una nueva clase de lipopéptidos semisintéticos, que inhiben la síntesis del 1,3-b glucano, polisacárido de la pared celular de muchos hongos patógenos. Por este mecanismo de acción, tienen potencial uso en combinación con otros antifúngicos. Las tres equinocandinas tienen propiedades farmacológicas similares, con potente acción fungicida, in vitro, sobre Candida spp, y potente acción inhibitoria sobre Aspergillus spp, con eficacia demostrada en animales. Lamentablemente son inactivos contra Zygomycetes ${ }^{54}$.

\section{Cirugía}

Debe ser agresiva y precoz. Se requiere debridamiento extenso con remoción de todo el tejido infectado y necrótico, drenando las colecciones líquidas y abscesos; si es necesario, deben repetirse los aseos quirúrgicos.

Con frecuencia la mucormicosis rinocerebral exige para su curación de la enucleación del globo ocular y evisceración del contenido necrótico de la órbita y palatectomía ${ }^{1,34,55}$.

El tratamiento quirúrgico de la mucormicosis cutánea debe apoyarse en biopsia rápida intraoperatoria para asegurar la obtención de bordes libres de micosis al efectuar las resecciones de tejidos ${ }^{26,28}$.

Dada la gravedad y el mal pronóstico de esta enfermedad se han propuesto otras terapias como: - Anfotericina B tópica, oxígeno hiperbárico ${ }^{1,2,34}$.

- Cirugía sinusal endoscópica: se plantea que sólo podría tener un rol en el diagnóstico inicial, pero en general no se recomienda como terapia definitiva, a pesar que hay algunos reportes de casos tratados en forma exitosa con este tipo de cirugía ${ }^{44}$. En este número de la revista publicamos, por separado, tres casos pediátricos de mucormicosis rinocerebral que recibieron un tratamiento quirúrgico de este tipo con resolución favorable de la infección ${ }^{18-20}$; tal vez la edad de estos pacientes establezca una diferencia conceptual importante para el manejo en niños de esta rara afección.

\section{Pronóstico}

Hasta hace 20 años el pronóstico de esta infección era uniformemente fatal, posteriormente ha mejorado, aunque mantiene alta mortalidad. La sobrevida depende de varios factores, a saber: edad del paciente, enfermedad de base, localización de la infección, oportunidad del diagnóstico, reversión de los factores de riesgo de base, el uso oportuno de antifúngicos sistémicos y el tratamiento quirúrgico precoz y agresivo ${ }^{1,55}$.

\section{Profilaxis}

Para un microorganismo tan ubicuo y a la vez de tan baja incidencia es difícil concebir una profilaxis exitosa en cualquier escenario ni parece prudente plantear una quimioprofilaxis primaria con antifúngicos. Algunos autores han practicado la fungoprofilaxis secundaria en pacientes oncológicos recuperados de mucormicosis y que 
deben retomar una quimioterapia aplasiante, de manera similar a como se recomienda en candidiasis profunda y en aspergilosis.

Se ha recomendado específicamente para el medio hospitalario el empleo de filtros de aire en las unidades de aislamiento para pacientes severamente inmunocomprometidos ${ }^{56}$. Una medida sencilla es evitar el uso de bajalenguas de madera en el examen de la mucosa oral en pacientes inmunocomprometidos, y en la inmovilización de las extremidades de neonatos prematuros.

\section{Resumen}

La mucormicosis es una infección infrecuente causada por hongos oportunistas que pertenecen al orden Mucorales, familia Mucoraceae, cuyas características son invasión vascular por hifas, lo que determina trombosis e infartos. En general afecta pacientes con enfermedades subyacentes y produce infecciones severas invasoras y a menudo fulminantes. Algunas condiciones de riesgo para contraer la mucormicosis son: cetoacidosis diabética, terapia inmunosupresora, leucemia y linfomas con neutropenia prolongada. Se describen varias presentaciones clínicas, según su localización anatómica, siendo la forma rinocerebral la más frecuente, especialmente en pacientes diabéticos. Aunque esta infección tiene una alta morbimortalidad, su pronóstico ha mejorado los últimos años, en base a medidas terapéuticas como la oportuna corrección de los factores predisponentes, uso de anfotericina $\mathrm{B}$ y una cirugía precoz y agresiva. Es muy importante sospechar esta infección en pacientes con factores predisponentes de manera de hacer un diagnóstico precoz.

\section{Bibliografía}

1.- Sugar A M. Chapter 249: Agents of mucormycosis and related. En Mandell G L, Bennett J E and Dolin R editors, Mandell, Douglas and Bennett's Principles and Practice of Infectious Diseases. Fifth edition. Churchill Livingstone, Philadelphia 2000; 2685-94.

2.- Wiedermann B L. Zygomycosis. En: Textbook of Pediatric Infectious Diseases, Ralph D. Feigin - James D. Cherry editors. $4^{\text {th }}$ edition, 1998. W.B.Saunders Company, Phhiladelphia. pp: 2354-60.

3.- Kwon-Chung K J, Bennett J E. Mucormycosis (Phycomycosis, Zygomycosis) En: Medical Mycology. Editorial Lea and Febiger 1992: 524-59

4.- Richardosn M D, Shankland G S. Chapter 98: Rhizopus, Rhizomucor, Absidia and Other Agents of Systemic and Subcutaneous Zygomycoses. Manual of Clinical Microbiology. Patrick R. Murray, Ellen Jo Baron, Michael Pfaller, Fred C. Tenover, Robert H. Yolken. 7th edition. ASM Washington DC 1997; 1242-58.

5.- Robertson A F, Joshi V V, Ellision D A, Cedars J C. Zygomycosis in neonates. Pediatr Infect Dis J 1997; 16: 812-5.

6.- Amin S B, Ryan R M, Metlay L A, Watson W J.
Absidia corymbifera infections in neonates. Clin Infect Dis 1998; 26: 900-2.

7.- Kontoyiannis D P, Wessel V C, Bodey G P, Rolston K V. Zygomycosis in the 1990 s in a tertiary-care cancer center. Clin Infect Dis 2000; 30: 851-6.

8.- Walsh T J, Pizzo P A. Nosocomial Fungal Infections: A classification for hospital-acquired fungal infections and mycoses arising from endogenous flora or reactivation. Ann Rev Microbiol 1988; 42: 517.

9.- Weems J J Jr, Davis B, Tablan O C, Kaufman L, Martone W J. Construction activity: an independent risk factor for invasive aspergillosis and zygomycosis in patients with hematologic malignancy. Infect Control 1987; 8: 71-5.

10.- Holzel H, Macqueen S, MacDonald A, Alexander S, Campbell C K, Jonson E M, Warnock D W. Rhizopus microsporus in wooden tongue depressors: a major threat or minor inconvenience? J Hosp Infect 1998; 38 (2): 113-8.

11.- Cox J, Corona S, Donoso S. Mucormicosis orbitaria y cerebral en dos pacientes diabéticos. Rev Méd Chile 1985; 113 (4): 341-4.

12.- Villarroel F. Mucormicosis orbitaria (ficomicosis, zigomicosis). Arch Chil Oftalmol 1985; 42 (2): 61-5.

13.- Oddó D, Lobos T, Riquelme R, Thompson L, Acuña G. Zigomicosis: Estudio anátomo-clínico y micológico de 17 casos. Rev Méd Chile 1987; 115: 314: 8.

14.- Gacitúa R, Chuecas F, Grebe G, Lira P, Rosenberg H. Hemoptisis masiva en un paciente inmunocomprometido. Bol Esc Med 1991; 20 (1): 66-9.

15.- Bravo M, Ferrer S, Rtchard M, Trujillo S. Mucormicosis rinocerebral. Comunicación de cuatro casos. Rev Méd Chile 1999; 127: 712-8.

16.- García P, Beltrán C, Guzmán AM, León P, Arredondo $\mathrm{M}$, Fonseca X. Diagnóstico rápido de dos casos de mucormicosis con tinción de blanco de calcoflúor. Rev Chil Infect 2001; 18: 285-90.

17.- Bertin H. Mucormicosis rinosinusal. Rev Otorrinolaringol Cir Cabeza Cuello 2003; 63: 122-6.

18.- Wicki M A, Borel A C, Villarroel C M, Cofré G J. Curación de mucormicosis rinocerebral en un paciente pediátrico con leucemia activa. Rev Chil Infect 2004; 21 (1): 48-52.

19.- Chávez P A y Rahal E M. Mucormicosis rinocerebral en un niño diabético. Tratamiento conservador. Rev Chil Infect 2004; 21 (1): 57-60.

20.- Spalloni M WMP, Glaser K P, Verdugo L P. Mucormicosis rinocerebral: sobrevida a largo plazo. Rev Chil Infect 2004; 21 (1): 53-56.

21.- Pamela Silva G P y Avilés L CL.Mucormicosis pulmonar. Rev Chil Infect 2004; 21 (1): 61-64.

22.- Artis W M, Fountain J A, Delcher H K, Jones H E. A mechanism of susceptibility to mucormycosis in diabetic ketoacidosis: transferring and iron availability. Diabetes 1982; 31: 1109-14.

23.- Boclaert J R, de Lochet M, Van Cutsen $J$ et al. Mucormycosis during desferoxamine therapy is a siderophore-mediated infection. In vitro and in vivo animal studies.J Clin Invest 1999; 91: 1979-86.

24.- Abe F, Inaba H, Katoh T, Hotchi M. Effects of iron and desferoxamine on Rhizopus infections. Mycopathologia 1990; 110: 87-91.

25.- Waldorf A R. Pulmonary defense mechanisms against opportunistic fungal pathogens. Immunol Sem 1989; 47: 243-71.

26.- Oh D, Notrica D. Primary cutaneous mucormycosis in infants and neonates: Case report and review of the literature. J Pediatr Surg 2002; 37: 1607-11. 
27.- Robertson A, Joshi V, Ellison D, Cedars J. Zygomycosis in neonates. Pediatric Infect Dis J 1997; 16: 812-5.

28.- Scheffler E, Miller G G, Classen D A. Zygomycosis infection of the neonate upper extremity. J Pediatr Surg 2003; 38: E21.

29.- Lee F Y, Mossad S B, Adal K A. Pulmonary mucormycosis: the last 30 years. Arch Intern Med 1999; 159: 1301-9.

30.- Pagano L, Ricci P, Tonso A et al. Mucormycosis in patients with haematological malignancies: a retrospective clinical study of 37 cases. GIMENA Infection Program. Br J Haematol 1997; 99 (2): 331-6.

31.- Brown R B, Johnson J H, Kessinger J M, Seally W C. Bronchovascular mucormycosis in the diabetic: an urgent surgical problem. Ann Thorac Surg. 1992; 53 (5): 854-5.

32.- Latif S, Saffarian N, Bellovich K, Provenzano R. Pulmonary mucormycosis in diabetic renal allograft recipients. Am J Kidney Dis 1997; 29 (2): 461-4.

33.- Nagy-Agren SE, Chu P, Smith G J, Waskin H A, Altice F L. Zygomycosis (mucormycosis) and HIV infection: report of three cases and review. J Acquir Immune Defic Syndr Hum Retrovirol 1995; 10 (4): 441-9. Review.

34.- González C, Rinaldi M, Sugar A. Zygomycosis. Infect Dis Clin North Am 2002; 16 (4): 895-914.

35.- Pagano L, Ricci P, Tonso A et al and the Gimema Infection Program. Fatal hemoptysis in pulmonary filamentous mycosis: An underevaluated cause of death in patient with acute leukaemia in haematological complete remission. A retrospective study and review of the literature. Br J Haematol 1995; 89: 500-5.

36.- Albeda S, Talbot G, Gerson S, Miller W, Cassileth P. Pulmonary cavitation and massive hemoptysis in invasive pulmonary aspergillosis. Am. Rev. Respir. Dis 1985; 131: 115-20.

37.- Robertson B, Bautista M, Russell T. Fibrosing mediastinitis secondary to zygomycosis in a 22 month old child. Pediatr Infect Dis Child J 2002; 21: 441-2.

38.- Leong A S Y. Granulomatous mediastinitis due to rhizopus species. Am J Clin Pathol 1978; 70:103-7.

39.- Tedder M, Spratt J, Anstadt M. Pulmonary mucormycosis: Results of medical and surgical therapy. Ann Thorac Surg 1994; 57: 1044-50.

40.- Potente G. CT findings in fungal opportunistic pneumonias: body and brain involvement. Comp Med Imaging Graph 1989; 13 (5): 423-8

41.- Glazer M, Nusair S, Breuer R, Lafair J, Sherman Y, Berkman N. The role of BAL in the diagnosis of pulmonary mucormycosis. Chest. 2000; 117 (1): 279-82.

42.- Earhart K C, Baugh W P. Rhinocerebral Mucormycosis. www.emedicine.com (update 10 enero, 2003) Accedido: 15/12/03.
43.- Diekema D J, Messer S A, Jones R N, Pfaller M A. Activities of caspofungin, posaconazol, ravuconazol, voriconazol and anphotericin B against 448 recent clinical isolates of filamentous fungi. J Clin Microbiol 2003; 41: 3623-6.

44.- Shah P D, Peters K R, Reuman P D. Recovery from rhinocerebral mucormycosis with carotid artery occlusion: a pediatric case and review of the literature. Pediatr Infect Dis J 1997; 16: 68-71.

45.- Parfrey N Z. Improved diagnosis and prognosis of mucormycosis. Medicine 1986; 65: 113-23.

46.- García-Díaz J B, Palau L, Pankey G A. Resolution of Rhinocerebral Zygomycosis associated with adjuvant administration of Granulocyte-Macrophage ColonyStimulating Factor. Clin Infect Dis 2001; 32: e16670.

47.- Casadevall A, Pirofski L. Adjunctive immune therapy for fungal infections. Clin Infect Dis 2001; 33: 104856.

48.- Barnert J, Behn W, Reich H. An amphotericin B resistant case of rhinocerebral mucormycosis. Infection 1985; 13: 48-50.

49.- Walsh T J, Seibel N L, Arndt C et al. Amphotericin B lipid complex in pediatric patients with invasive fungal infections. Pediatr Infect Dis J 1999; 18: 702-8.

50.- Sun Q N, Fother hill A W, McCarthy D I, Rinaldi M G, Graybill J R. In vitro activities of posaconazole, voriconazole, amphotericin $\mathrm{B}$, and fluconazole against 37 clinical isolates of Zygomycetes. Antimicrob Agents Chemother 2002; 46 (5): 1581-2.

51.- Dannaoui E, Meletiadis J, Mouton J W, Meis J FGM, Verwirj $\mathrm{P}$ E and The Eurofung Network. In vitro susceptibilities of zygomycetes to conventional and new antifungals. J Antimicrob Chemother 2003; 51: 45-52.

52.- Tobon A M, Arango M, Fernández D, Restrepo A. Mucormycosis (zygomycosis) in a heart-kidney transplant recipient: recovery after posaconazole therapy. Clin Infect Dis 2003; 36: 1488-91.

53.- Pfaller M A, Mecer S A, Boyken L, Hollis R J, Diekema D J. Posaconazole is a new triazole with in vitro and in vivo activity against zygomycosisDiag Microbiol Infect Dis 2003; 45: 241-4.

54.- Groll A H, Gea-Banacloche J C, Glasmacher A, JustNuebling G, Marschmeyer G, Walsch T J L. Clinical pharmacology of antifungal compounds. Infect Dis Clin North Am 2003; 17 (1): 159-91.

55.- Hamilton J F, Bartkowski H B, Rock J P. Management of CNS mucormycosis in the pediatric patient. Pediatr Neurosurg 2003; 38: 212-5.

56.- Lueg E A, Ballagh R H, Forte V. Analysis of the recent cluster of invasive fungal sinusitis at the Toronto Hospital for Sick Children. J Otorynol 1996; 25: 36670.

Correspondencia a:

W. María Pía Spalloni Macari

E-mail: wandapia@vtr.net 